\title{
Preferência por hospedeiro e estratificação de Culicidae (Diptera) em área de remanescente florestal do Parque Regional do Iguaçu, Curitiba, Paraná, Brasil ${ }^{1}$
}

\author{
Ana Cristina Tissot ${ }^{2} \&$ Mário Antonio Navarro-Silva ${ }^{2,3}$ \\ ${ }^{1}$ Contribuição número 1462 do Departamento de Zoologia, Universidade Federal do Paraná. \\ ${ }^{2}$ Departamento de Zoologia, Universidade Federal do Paraná. Caixa Postal 19020, 81531-980 Curitiba, Paraná, Brasil. E- \\ mail: mnavarro@ufpr.br; anatissot@ufpr.br \\ ${ }^{3}$ Bolsista de produtividade em pesquisa do CNPq.
}

\begin{abstract}
Host preference and Culicidae stratification in area of degradated inside forest of Regional do Iguaçu Park, Curitiba, Paraná, Brazil. Parks and plazas (green areas or vegetation islands) within urban areas can provide conditions for the development of populations of mosquitoes, many species of which are very adaptable to a variety of environments. The species of mosquitoes in the family Culicidae with animal hosts, in a vegetation island within an urban area, in Curitiba, in the state of Paraná, in southern Brazil was investigated. To examine whether these green areas contribute to urban populations, mosquitoes were collected five times each season (spring, summer and fall), from September 2000 to July 2001. CDC-M traps were set at two heights $(1.5 \mathrm{~m}$ and 6 m). A mammal (quinea pig, Cavia porcellus Linnaeus, 1758, Rodentia, Cavidae) and bird (Nothura maculosa (Temminck, 1815), Tinamiformes, Tinamidae) were used as bait. Traps were active from 16:00 to 20:00 h, with samples taken every 30 minutes. A total of 1,415 individual mosquitoes were collected, in 9 genera and 11 species, with $44 \%$ of the individuals taken in Spring samples, $40 \%$ in Summer and $16 \%$ in Fall samples. The $1.5 \mathrm{~m}$ traps with mammal bait captured the most mosquitoes ( $41 \%)$, with the $6 \mathrm{~m}$ trap with bird bait being second $(30 \%)$, followed by $1.5 \mathrm{~m}$ and bird bait (19\%) and 6m and mammal bait (10\%). Mansonia (Mansonia) fonsecai (Pinto, 1932) and Mansonia (Mansonia) pessoai (Barreto e Coutinho, 1944) was the most common species found with both baits. Other species captured were: Mansonia (Mansonia) wilsoni (Barreto \& Coutinho, 1944); Mansonia (Mansonia) titillans (Walker, 1848); Culex (Culex) acharistus Root, 1927; Culex (Culex) sp.; Wyomyia (Phoniomyia) quasilongirostris Theobald, 1907; Ochlerotatus (Ochlerotatus) scapularis (Rondani, 1848); Psorophora Oanthinosoma) ferox (Humboldt, 1819); Culex (Melanoconion) sp.; Coquillettidia (Rhynchotaenia) venezuelensis (Theobald, 1912); Aedeomyia (Aedeomyia) squamipennis (Lynch Arribálzaga, 1878); Ochlerotatus (Ochlerotatus) serratus (Theobald, 1901); Limatus durhami Theobald, 1901 and Aedes (Stegomyia) albopictus (Skuse, 1894). This last species has a great epidemiological importance. This study shows that green areas (vegetation islands) within urban centers can be an important source of potentially epidemiological mosquitoes.

KEY WORDS. Animal bait, CDC trap, Culicidae, ecology, Mansonia.
\end{abstract}

RESUMO. A investigação das populações de Culicidae em áreas de remanescentes florestais inseridas em área urbana, podem fornecer subsídios para compreensão dos processos de utilização de habitats apresentando diferentes graus de interferência antrópica. Foram investigadas espécies potencialmente zoofílicas durante o período vespertino no interior de remanescente florestal, no espaço urbano de Curitiba, Paraná. Durante o período de setembro de 2000 a junho de 2001, foram realizadas cinco coletas por estação, com auxílio de armadilhas CDC-M instaladas em dois estratos verticais, a 1,5 $\mathrm{m}$ do solo e na copa das árvores $(6 \mathrm{~m})$. Como iscas foram utilizados mamíferos Cavia porcellus (Linnaeus, 1758) (Rodentia, Cavidae) e aves Nothura maculosa (Temminck, 1815) (Tinamiformes, Tinamidae) em cada um dos estratos, com revezamento das iscas animais. As armadilhas foram operadas no intervalo horário das 16:00 às 20:00 h, sendo retiradas amostras a cada intervalo de 30 minutos. Em 60 horas de operação das armadilhas CDC-M, foram capturados 1.407 exemplares de Culicidae, sendo 1.143 espécies identificadas, distribuídas em nove gêneros e 13 espécies. As espécies mais freqüentes foram Mansonia(Mansonia) fonsecai (Pinto, 1932) e Mansonia (Mansonia) pessoai (Barreto e Coutinho, 1944), destaca-se também a ocorrência de: Ochlerotatus (Ochlerotatus) scapularis (Rondani, 1848); Psorophora (Janthinosoma) ferox (Humboldt, 1819) e Aedes (Stegomyia) albopictus (Skuse, 1894). Na área foram detectadas a presença de espécies com graus variados de importância epidemiológica e com tendência a explorar ambientes exófilos, florestais e peridomiciliares.

PALAVRAS CHAVE. Armadilha CDC, isca animal, Culicidae, ecologia, Mansonia. 
A atuação antrópica modificando o ambiente natural e o processo de urbanização podem proporcionar condições favoráveis à proliferação de Culicidae, considerando também a capacidade desta família de dípteros em adaptarem-se às diversas alterações ambientais, pode-se esperar mudanças em relação aos quadros epidemiológicos das infecções transmitidas pelas espécies vetoras de doenças (ForatTINI et al.1998).

A investigação da fauna de Culicidae em ambientes com predomínio de condições naturais, porém sob forte influência do homem em razão de sua inserção no cenário urbano, pode revelar a presença de espécies com importância epidemiológica.

Em uma área dentro do ecossistema urbano da Grande São Paulo, Parque Ecológico do Tietê, onde são admitidos e mantidos diversos grupos de vertebrados, Pereira et al. (2001) e TAIPE-LAgos \& NATAL (2003), consideraram o ambiente como tendo fatores de risco para a introdução e manutenção de arbovírus, como o vírus Ilhéus, devido à presença de culicídeos potencialmente vetores no local.

No município de Curitiba alguns trabalhos já abordaram a ecologia de Culicidae, em áreas de vegetação, presentes no ecossistema urbano, que sofrem ou sofreram ação humana com variados graus de intensidade. Si lva \& Lozoveı (1996, 1998, 1999) identificaram espécies que apresentam diferentes níveis de domiciliação eimportância epidemiológica, como por exemplo: Ochlerotatus (Ochlerotatus) scapularis (Rondani, 1848), considerada de ambiente alterado e Haemagogus (Conopostegus) leucocelaenus (Dyar \& Shannon, 1924) que explora preferencialmente ambiente silvestre, apresentando relevante papel na epidemiologia da febre amarela. Também detectaram a presença de Culex (Culex) quinquefasciatus Say, 1823, espécie com elevado grau de domiciliação e apontada para região neotropical como o mais eficiente vetor da filariose bancroftiana ao homem (ForatTINI 2002).

A armadilha CDC (Center of Disease Control) e particularmente eficiente na análise quantitativa e qual itativa de populações de Culicidae, em áreas naturais, em diferentes estratos vegetais, ou alteradas pela atividade antrópica. O emprego da armadilha CDC mostrou-se eficiente, na captura de espécies de Culicidae, para monitoramento de potenciais vetores defilariose, arbovirose, malária e conhecimento da fauna culicidiana, em al guns casos obtiveram el evado rendimento nas capturas quando a armadilha foi suplementada com atrativo de $\mathrm{CO}_{2}$, gelo seco, ácido láctico ou isca animal (Christensen \& ANDreWs 1976, Zaim et al. 1986, Forattini et al. 1989, MARQuetti et al. 1990, VASCONCELos et al. 1991, GILLILAND et al. 1995, MACIÁ 1996, GAD et al. 1999).

Em estudo específico para determinar a fauna de Culicidae, Gomes et al. (1987) utilizando armadilha CDC com isca animal (pombo e roedor), obtiveram resultados satisfatórios para Anopheles cruzii Dyar \& Knab,1908.

Em decorrência da ausência de trabal hos, desenvolvidos em fragmentos de mata inserida na área urbana de Curitiba, utilizando armadilha CDC acompanhada de isca animal para estudo da fauna de Culicidae, o presente estudo tem por obje- tivo acrescentar informações sobre a atividade de mosquitos em hospedeiros representados por aves e mamíferos em diferentes estratos em é área de fragmento florestal contíguo ao Zoológico Municipal de Curitiba.

\section{MATERIAL E MÉTODOS}

O trabalho foi desenvolvido em área de mata do Parque Regional do Iguaçu, pertencente ao município de Curitiba que se situa na porção centro-sul do primeiro planalto do Estado do Paraná, na porção oriental do Estado nas coordenadas 2525'04"Se 49이'30"W, a uma altitude de 945 m (M AACK 1981).

$A$ área de mata estudada é caracterizada como um remanescente de floresta ombrófila mista (floresta com Araucaria) com três estratos bem definidos, o dossel, com indivíduos entre 16 e $22 \mathrm{~m}$ de altura, onde se encontra a espécie Araucaria angustifolia (Araucariaceae), que não supera $22 \mathrm{~m}$ de altura; o intermediário ou sub-bosque, com espécies típicas como a guassatunga (Casearia sylvestris, Flacourtiaceae), a erva-mate (Ilex paraguariense, Aquifoliaceae), a falsa-espinheira-santa (Sorocea bonplandii, Moraceae) entre outras e o arbustivo-herbáceo representado por diversas espécies de samambaias (Pteridophyta), agrupamentos de caetê (Marantaceae) e inúmeras espécies de gramíneas (Poaceae).

Pouco distante, mas ainda no interior dos domínios do Parque Iguaçu, existem lagos de diferentes dimensões que no passado foram utilizados para extração pretérita de areia (cavas), com predomínio das espécies aquáticas: alface-d'água (Pistia stratiotes, Araceae), lentilhas-d'água (Lemnaceae), ervade-sapo (Salvinia auriculata, Salviniaceae), aguapé (Eichornia crassipes, Pontederiaceae), hortelã-do-brejo (Heteranthera reniformis, Pontederiaceae), rainha-dos-lagos (Pontederia cordata, Pontederiaceae), taboa (Typha domingensis, Typhaceae) ou capim-capivara (Echynochloa polystachya, Poaceae).

Quanto ao aspecto climático, durante o período de 10 meses de coleta compreendido entre setembro de 2000 e junho de 2001, a média das temperaturas mínima, média e máxima atingiram respectivamente $14,9^{\circ} \mathrm{C}, 18,9{ }^{\circ} \mathrm{C}$ e $24,6{ }^{\circ} \mathrm{C}$. A média da umidade relativa mínima, média e máxima foi de $56,7 \%, 79 \%$ e $93,4 \%$, respectivamente. Enquanto a precipitação pluviométrica acumulada para o período foi de $1262,2 \mathrm{~mm}$, com média diária de aproximadamente 4,65 mm.

Em cada estação durante todo o período de coleta, a mé dia de temperatura mais el evada foi de 28,1 으 C durante o verão e a mais baixa foi de $13,7 \circ$ C durante o outono, a média da umidade relativa do ar ficou em $93,7 \%$ no outono e 53,4\% na primavera e em relação à precipitação pluviométrica obteve-se a mé dia de 7,0 mm³ no verão com uma somatória de $625,2 \mathrm{~mm}^{3}$. Estes dados, assim como aquel es utilizados para comparação com as populações de Culicidae foram obtidos junto a Estação do Simepar (Sistema M eteorológico do Estado do Paraná), Iocalizada no Centro Politécnico, Universidade Federal do Paraná (2526'S e 4916'W, a uma altitude de 923,50 m). 
As capturas de Culicidae foram realizadas no período de setembro de 2000 a junho de 2001, utilizando quatro armadiIhas modificadas, CDC-M descrito por NATAL et al. em 1991, a partir do modelo prático e desmontável descrita por Gomes et al. (1985) e Sudia \& ChamberLain (1962), com objetivo de obtenção de grande número de exemplares de Culicidae para isolamento de arbovírus, iscadas com atrativo animal, ave (Nothura maculosa (Temminck, 1815), Tinamiforme, Tinamidae) e mamífero (Cavia porcellus (Linnaeus, 1758), Rodentia, Cavidae) ambos de pequeno porte. As armadilhas foram instaladas, duas a 1,5 $\mathrm{m}$ do solo e duas na copa das árvores, aproximadamente $6 \mathrm{~m}$, em estratificação vertical em dois pontos no interior da mata, com uma distância de aproximadamente $7 \mathrm{~m}$ de um ponto a outro. No primeiro, a ave na primeira altura e o mamífero na segunda, invertendo-se a posição das iscas no segundo ponto. Ao total foram realizadas 15 coletas sendo cinco em cada uma das estações, primavera, verão e outono.

As amostragens de culicídeos foram efetuadas no período vespertino, a cada 30 minutos, com início às 16:00 h e término às 20:00 h. Em seqüência os exemplares foram levados até o laboratório de Entomologia Médica e Veterinária do Departamento deZoologia da Universidade Federal do Paraná, para identificação. Parte dos exemplares encontra-se depositado na Coleção de Entomologia Padre Jesus Santiago Moure, Departamento de Zoologia, Universidade Federal do Paraná (DZUP).

Para análise dos dados obtidos através das capturas foi utilizada uma análise paramétrica de correlação. Procuramos detectar a forma de correlação entre o número de mosquitos capturados nas diferentes armadi lhas com os fatores abióticos: temperatura $(\stackrel{\circ}{ } \mathrm{C})$, umidade relativa do ar (\%) e precipitação pluviométrica (mm). Nas correlações significativas ou não, foi real izada anál ise de regressão, testando-se três model os (linear, exponencial e potencial).

Análises de regressão linear, exponencial e potencial foram realizadas para a obtenção do coeficiente de determinação $\left(r^{2}\right)$ que nos informa a porcentagem da variação de y que é explicada pela variação de $x$.

Para o rendimento de Culicidae, comparando as estações com a estratificação das armadilhas, as estações com as iscas utilizadas e as iscas com as estratificações, foram aplicados os testes de Levene de Homogeneidade e em seguida o teste de Análise de Variância ao nível de significância de 0,05.

\section{RESULTADOS E DISCUSSÃ O}

$\mathrm{Na}$ área do fragmento florestal do Parque Regional do Iguaçu, em 60 horas de operação das armadilhas CDC-M, foram capturados 1.407 exemplares de Culicidae, sendo 1.143 espécimes identificadas, em média 23,5 por hora, pertencentes a nove gêneros totalizando 13 espécies. Sendo a espécie predominante Mansonia (Mansonia) fonsecai (Tabs I, II e III).

O número total de mosquitos coletados nas armadilhas CDC-M iscadas com mamíferos foi 721 (51\%), superior ao coletado nas armadilhas iscadas com aves 686 (48\%). Entretanto houve uma variação mais acentuada, na freqüência de capturas, entre os estratos verticais nas armadilhas iscadas com mamíferos do que nas armadilhas com iscas representadas por aves (Tabs II e III, Fig. 1).

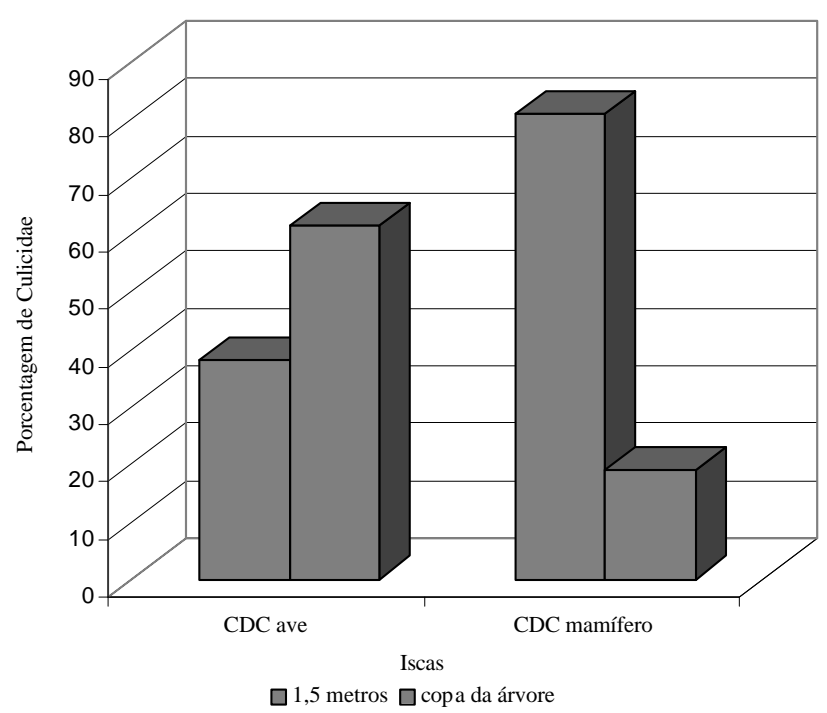

Figura 1. Porcentagem do total de Culicidae coletados relacionando dois tipos de iscas utilizadas, mamífero e ave, nas armadiIhas CDC-M a 1,5 e $6 \mathrm{~m}$ de altura, de setembro de 2000 a junho de 2001, em área de fragmento florestal, do Parque Regional do Iguaçu, Curitiba, Paraná.

Comparando os dois conjuntos de armadilhas, o primeiro (Tab. II) representado pelas armadilhas CDC-M com ave a 1,5 m do solo e mamífero na copa da árvore e o segundo conjunto (Tab. III) representado pela inversão da posição da ave e mamífero junto às armadilhas CDC-M, observamos que neste segundo conjunto foram capturados um número superior de Culicidae em relação ao primeiro conjunto.

Na comparação entre o rendimento total de Culicidae capturados nas armadilhas com aves e mamíferos, não foi detectado diferença significativa ( $p=0,916$ e $F=0,011$ ). No entanto, o rendimento de mosquitos capturados não foi equilibrada na comparação entre a posição da isca no estrato vertical. Na copa da árvore houve predomínio de mosquitos capturados na CDC-M com ave com a diferença sendo significativa em relação a CDC-M com mamífero no mesmo estrato ( $p=$ 0,049 e $F=4,212$ ). No estrato inferior a diferença entre as armadilhas com aves e mamíferos não foi significativa ( $p=0,456$ e $F=0,570$ ) (Tab. IV).

Analisando o rendimento total de mosquitos em relação aos três períodos estacionais, primavera, verão e outono, observamos que não houve diferença significativa entre as estações nas armadilhas com aves ( $p=0,218$ e $F=1,705$ ) e mamífe- 
Tabela I. Resenha das espécies de Culicidae atraídas (X) ou não (-) pelas iscas animais colocadas junto das armadilhas modificadas, CDC-M, durante o período de setembro de 2000 a junho de 2001, em área de remanescente florestal, do Parque Regional do Iguaçu, Curitiba, Paraná.

\begin{tabular}{|c|c|c|c|c|}
\hline \multirow{3}{*}{ Espécies } & \multicolumn{4}{|c|}{ CDC-M } \\
\hline & \multicolumn{2}{|c|}{ Ave } & \multicolumn{2}{|c|}{ Mamífero } \\
\hline & $1,5 \mathrm{~m}$ & Copa & $1,5 \mathrm{~m}$ & Copa \\
\hline Culex (Culex) acharistus Root, 1927 & $\mathrm{x}$ & $\bar{X}$ & $\mathrm{x}$ & $\mathrm{X}$ \\
\hline Mansonia (Mansonia) titillans (Walker, 1848) & $x$ & $x$ & $x$ & $x$ \\
\hline Mansonia (Mansonia) wilsoni (Barretto \& Coutinho, 1944) & $x$ & $\mathrm{x}$ & $x$ & $x$ \\
\hline Mansonia (Mansonia) fonsecai (Pinto, 1932) & $x$ & $x$ & $x$ & $x$ \\
\hline Mansonia (Mansonia) pessoai (Barretto \& Coutinho, 1944) & $\mathrm{x}$ & $\mathrm{x}$ & $\mathrm{x}$ & $x$ \\
\hline Ochlerotatus (Ochlerotatus) scapularis (Rondani, 1848) & $x$ & $\mathrm{x}$ & $\mathrm{x}$ & $x$ \\
\hline Psorophora (Janthinosoma) ferox (Humboldt, 1819) & $\mathrm{X}$ & - & $\mathrm{x}$ & $x$ \\
\hline Coquillettidia (Rhynchotaenia) venezuelensis (Theobald,1912) & - & $\mathrm{x}$ & $x$ & $x$ \\
\hline Wyomyia (Phoniomyia) quasilongirostris Theobald, 1907 & - & - & $\mathrm{x}$ & $x$ \\
\hline Ochlerotatus (Ochlerotatus) serratus (Theobald, 1901) & $\mathrm{x}$ & - & $\mathrm{x}$ & - \\
\hline Aedeomyia (Aedeomyia) squamipennis (Lynch Arribálzaga, 1878) & - & $\mathrm{X}$ & - & $\mathrm{X}$ \\
\hline Limatus durhami Theobald, 1901 & $\mathrm{x}$ & - & - & - \\
\hline Aedes (Stegomyia) albopictus (Skuse, 1894) & $x$ & - & - & - \\
\hline
\end{tabular}

Tabela II. Total das espécies de Culicidae coletadas no mesmo ponto, nas armadilhas iscadas com ave a 1,5 m e mamífero na copa da árvore, de acordo com as estações do ano, de setembro de 2000 a junho de 2001, em área de remanescente florestal, do Parque Regional do Iguaçu, Curitiba, Paraná.

\begin{tabular}{|c|c|c|c|c|c|c|c|c|c|}
\hline \multirow{2}{*}{ Espécies } & \multicolumn{4}{|c|}{ CDC-M ave $1,5 \mathrm{~m}$} & \multicolumn{4}{|c|}{ CDC-M mamífero copa } & \multirow{2}{*}{ Total } \\
\hline & Primavera & Verão & Outono & Total & Primavera & Verão & Outono & Total & \\
\hline Mansonia (M.) fonsecai & 40 & 39 & 11 & 90 & 15 & 59 & 7 & 81 & 171 \\
\hline Mansonia (M.) pessoai & 36 & 3 & 12 & 51 & 13 & 4 & 6 & 23 & 74 \\
\hline Culex (C.) acharistus & 17 & 33 & 3 & 53 & 1 & 3 & 1 & 5 & 58 \\
\hline Mansonia (M.) wilsoni & 8 & 7 & 20 & 35 & 1 & 0 & 3 & 4 & 39 \\
\hline Mansonia (M.) titillans & 2 & 1 & 1 & 4 & 0 & 0 & 2 & 2 & 6 \\
\hline Ochlerotatus (0.) scapularis & 0 & 1 & 0 & 1 & 0 & 3 & 2 & 5 & 6 \\
\hline Coquillettidia (R.) venezuelensis & 0 & 0 & 0 & 0 & 0 & 2 & 0 & 2 & 2 \\
\hline Aedes (S.) albopictus & 1 & 0 & 1 & 2 & 0 & 0 & 0 & 0 & 2 \\
\hline Psorophora (J.) ferox & 0 & 1 & 0 & 1 & 0 & 1 & 0 & 1 & 2 \\
\hline Aedeomyia (A.) squamipennis & 0 & 0 & 0 & 0 & 0 & 1 & 0 & 1 & 1 \\
\hline Limatus durhami & 0 & 0 & 1 & 1 & 0 & 0 & 0 & 0 & 1 \\
\hline Ochlerotatus $(0$.$) serratus$ & 1 & 0 & 0 & 1 & 0 & 0 & 0 & 0 & 1 \\
\hline Wyomyia (P.) quasilongirostris & 0 & 0 & 0 & 0 & 0 & 1 & 0 & 1 & 1 \\
\hline Total & 105 & 85 & 49 & 239 & 30 & 74 & 21 & 125 & 364 \\
\hline
\end{tabular}

$\operatorname{ros}(p=0196$ e $F=1,866)$. Quando isolamos o rendimento por estação confrontando os dois tipos de isca, encontramos diferença significativa apenas durante o outono $(p=0,037$ e $F=$ 6,184), entre mamíferos e aves na copa das árvores (Tab. IV).

Em relação aos dados de temperatura, umidade relativa do ar e precipitação pluviométrica, observamos que os valores do coeficiente de determinação $\left(r^{2}\right)$ indicam reduzida influência desses fatores no rendimento das capturas de mosquitos, independente da forma como os dados foram analisados, mesmo observando os diferentes períodos detempo imediatamente anterior a execução das capturas. (Tabs VI, VII e VIII).

Em relação ao horário de captura, a partir das 18:00 ho-

Revista Brasileira de Zoologia 21(4): 877-886, dezembro 2004 
Tabela III. Total das espécies de Culicidae coletadas no mesmo ponto, nas armadilhas iscadas com ave na copa da árvore e mamífero a 1,5 m, de acordo com as estações do ano, de setembro de 2000 a junho de 2001, em área de remanescente florestal, do Parque Regional do Iguaçu, Curitiba, Paraná

\begin{tabular}{|c|c|c|c|c|c|c|c|c|c|}
\hline \multirow{2}{*}{ Espécies } & \multicolumn{4}{|c|}{ CDC-M mamífero $1,5 \mathrm{~m}$} & \multicolumn{4}{|c|}{ CDC-M ave copa } & \multirow{2}{*}{ Total } \\
\hline & Primavera & Verão & Outono & Total & Primavera & Verão & Outono & Total & \\
\hline Mansonia (M.) fonsecai & 69 & 137 & 24 & 230 & 47 & 91 & 27 & 165 & 395 \\
\hline Mansonia (M.) pessoai & 114 & 11 & 17 & 142 & 13 & 4 & 16 & 33 & 175 \\
\hline Culex (C.) acharistus & 0 & 1 & 0 & 1 & 13 & 71 & 21 & 105 & 106 \\
\hline Mansonia (M.) wilsoni & 6 & 6 & 10 & 22 & 16 & 6 & 5 & 27 & 49 \\
\hline Wyomyia (P.) quasilongirostris & 0 & 14 & 2 & 16 & 0 & 0 & 0 & 0 & 16 \\
\hline Ochlerotatus (0.) scapularis & 2 & 9 & 0 & 11 & 1 & 0 & 1 & 2 & 13 \\
\hline Psorophora (J.) ferox & 0 & 9 & 0 & 9 & 0 & 0 & 0 & 0 & 9 \\
\hline Mansonia (M.) titillans & 1 & 3 & 1 & 5 & 1 & 1 & 1 & 3 & 8 \\
\hline Coquillettidia (R.) venezuelensis & 0 & 2 & 0 & 2 & 0 & 2 & 1 & 3 & 5 \\
\hline Ochlerotatus (0.) serratus & 2 & 0 & 0 & 2 & 0 & 0 & 0 & 0 & 2 \\
\hline Aedeomyia (A.) squamipennis & 0 & 0 & 0 & 0 & 0 & 1 & 0 & 1 & 1 \\
\hline Total & 194 & 192 & 54 & 440 & 91 & 176 & 72 & 339 & 779 \\
\hline
\end{tabular}

Tabela IV. Descrição das análises de variância, comparando o rendimento de Culicidae capturados com os dados estacionais e as armadilhas CDC-M, durante o período de setembro de 2000 a junho de 2001, em área de remanescente florestal, do Parque Regional do Iguaçu, Curitiba, Paraná ( $p \leqslant 0,05$ e $F \leqslant 0,05$ ).

\begin{tabular}{|c|c|c|c|c|c|c|}
\hline & \multicolumn{2}{|c|}{$1,5 \mathrm{~m}$ do solo } & \multicolumn{2}{|c|}{ Copa da árvore } & \multicolumn{2}{|c|}{ Total } \\
\hline & $\mathrm{F}$ & $\mathrm{P}$ & $\mathrm{F}$ & $\mathrm{P}$ & $\mathrm{F}$ & $P$ \\
\hline Mamífero X Ave Primavera & 0,500271 & 0,499463 & 2,374646 & 0,161888 & 0,132705 & 0,725084 \\
\hline Mamífero X Ave Verão & 2,108014 & 0,184591 & 1,613429 & 0,239706 & 0,002600 & 0,960585 \\
\hline Mamífero X Ave Outono & 0,033457 & 0,859417 & 6,182135 & 0,037734 & 1,170701 & 0,310790 \\
\hline Ave $X$ estações & 1,101474 & 0,363761 & 0,683517 & 0,523452 & 1,705633 & 0,218771 \\
\hline Mamífero X estações & 1,017341 & 0,390726 & 3,007772 & 0,087338 & 1,866709 & 0,196856 \\
\hline Ave $\mathrm{X}$ mamífero & 0,570629 & 0,456317 & 4,212326 & 0,049585 & 0,011274 & 0,916198 \\
\hline
\end{tabular}

Tabela V. Descrição das análises de variância, comparando o rendimento de Culicidae capturados com os dados estacionais e as armadilhas CDC-M, durante o período de setembro de 2000 à junho de 2001, em área de remanescente florestal, do Parque Regional do Iguaçu, Curitiba, Paraná $(p \leqslant 0,05$ e $F \leqslant 0,05)$.

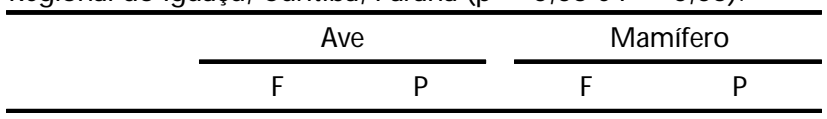

\begin{tabular}{lllll} 
Copa X Solo & 1,719789 & 0,200373 & 6,570483 & 0,016602 \\
\hline
\end{tabular}

ras observamos aumento na atividade dos Culicidae, no interior do fragmento florestal, independente do estrato e da isca utilizada na CDC-M, com discreto aumento na armadilha com mamífero na copa da árvore (Figs 2 e 3).

O principal gênero de Culicidae capturado foi Mansonia Blanchard, 1901 com aproximadamente 74\% (1043 espécimens) do total, com maior ocorrência na primavera (48\%) seguida do verão (36\%) e outono ( $15 \%$ ), este comportamento não se repete de forma completa para cada uma das espécies. Quando reunimos os números absolutos das quatro espécies em ordem decrescente, Mansonia fonsecai (Pinto, 1932), Mansonia pessoai (Barreto \& Coutinho, 1944), Mansonia wilsoni (Barreto \& Coutinho, 1944), Mansonia titillans (Walker, 1848), observamos que $55 \%$ dos exemplares destas espécies foram capturadas nas armadilhas com mamíferos e analisando o estrato independente do tipo de isca $63 \%$ dos exemplares foram capturados a $1,5 \mathrm{~m}$ do solo.

A espécie predominante Mansonia fonsecai ocorreu com maior freqüência durante o verão em armadilhas iscadas com aves e mamíferos independente do estrato (Figs 5 e 6). Quando analisamos o comportamento desta espécie em relação a isca observamos que aquela representada por mamífero exerce maior atração do que a ave. Quando se associa o tipo de isca e estrato, observamos que as aves exercem maior atração para aquela espécie quando está na copa, enquanto que o mamífe- 

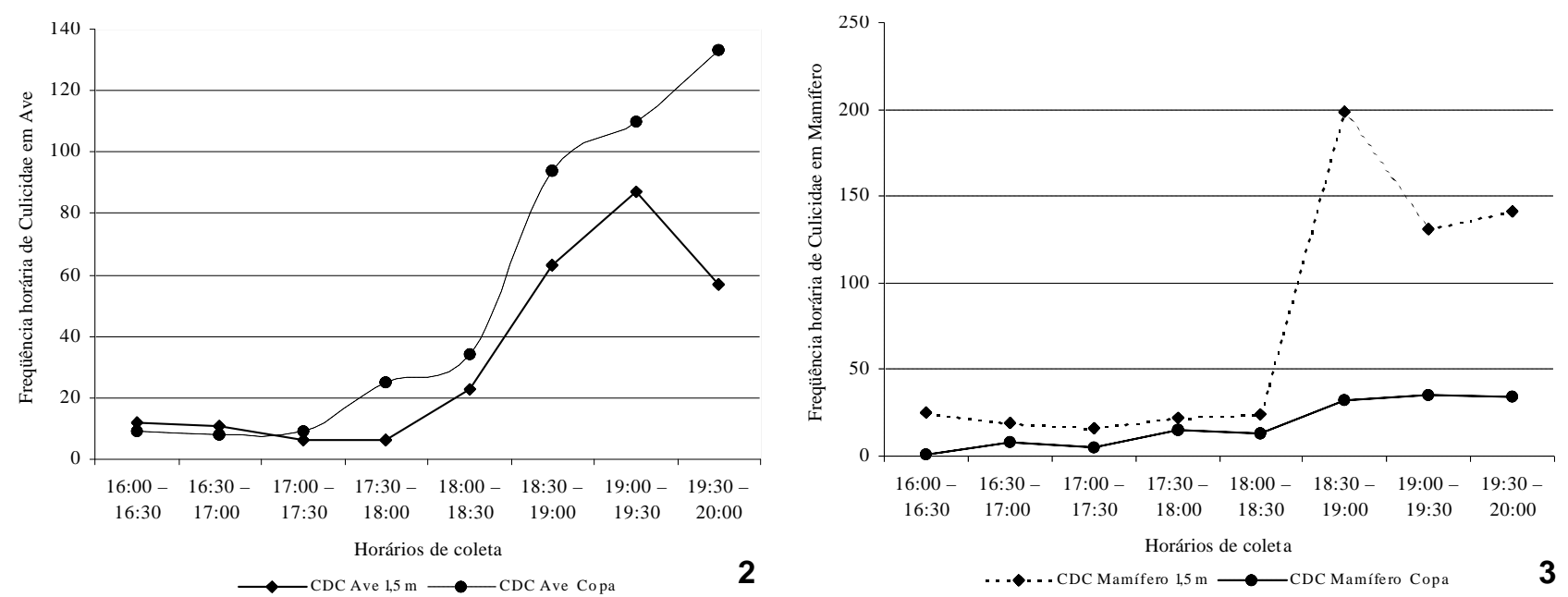

Figuras 2-3. Freqüência horária de Culicidae comparando os dois estratos de armadilhas CDC iscada com ave a 1,5 m e na copa da árvore (2) e mamífero a 1,5 m e na copa da árvore (3), de setembro de 2000 a junho de 2001, das 16:00 às 20:00 horas, em área de fragmento florestal do Parque Regional do Iguaçu, Curitiba, Paraná.

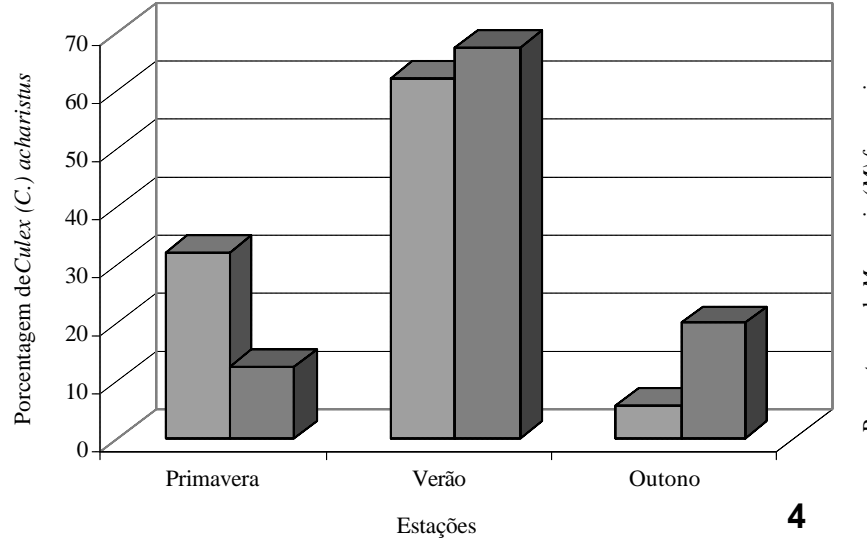

$\square 1,5 \mathrm{~m}$ do solo $\square$ Copa da árvore

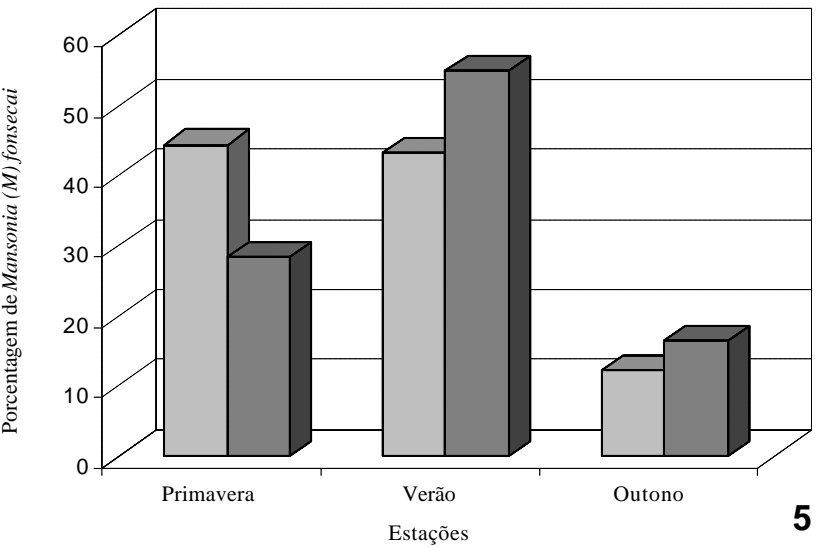

$\square 1,5 \mathrm{~m}$ do solo $\square$ Copa da árvore

Figuras 4-5. Porcentagem de Culex (Culex) acharistus (4) e M ansonia (Mansonia) fonsecai (5) coletada na armadilha CDC-M iscada com ave, de setembro de 2000 a junho de 2001, em área de remanescente florestal do Parque Regional do Iguaçu, Curitiba, Paraná.

ro quando está no solo, chegando a diferença no caso da ave e mamífero de aproximadamente duas e três vezes respectivamente (Tabs II e III).

Mansonia pessoai, embora com números inferiores ao registrado para Mansonia fonsecai, apresentou comportamento semelhante ao observado para esta espécie em relação as iscas utilizadas, diferindo quanto ao período estacional, ocorrendo preferencialmente durante a primavera (Fig. 7). Mansonia wilsoni demonstrou maior preferência pelas armadilhas iscadas com aves, independente do estrato analisado com número aproximado para primavera e outono. Já Mansonia titillans, apresentou número reduzido em todas as armadilhas, independente da isca e do estrato (Tabs II e III).
O elevado número de Mansonia no interior do fragmento de mata, no caso específico do Parque Regional do Iguaçu pode ser atribuída as condições ambientais das imediações, onde encontramos um grande número de lagoas ou cavas perenes com abundante cobertura vegetal aquática flutuante. As macrófitas, local de desenvolvimento dos imaturos deMansonia, podemos considerar como ocupando posição ambival ente em ambientes aquáticos, sendo necessárias para um grande número de espécies de animais associadas a elas, disponibilizando abrigo e alimentação. Por outro lado, servem de suporte para espécies de Mansonia potencialmente vetoras de arbovírus.

Podemos também relacionar o número el evado a mobilidade entre os criadouros e a busca pelo hospedeiro. De acor- 

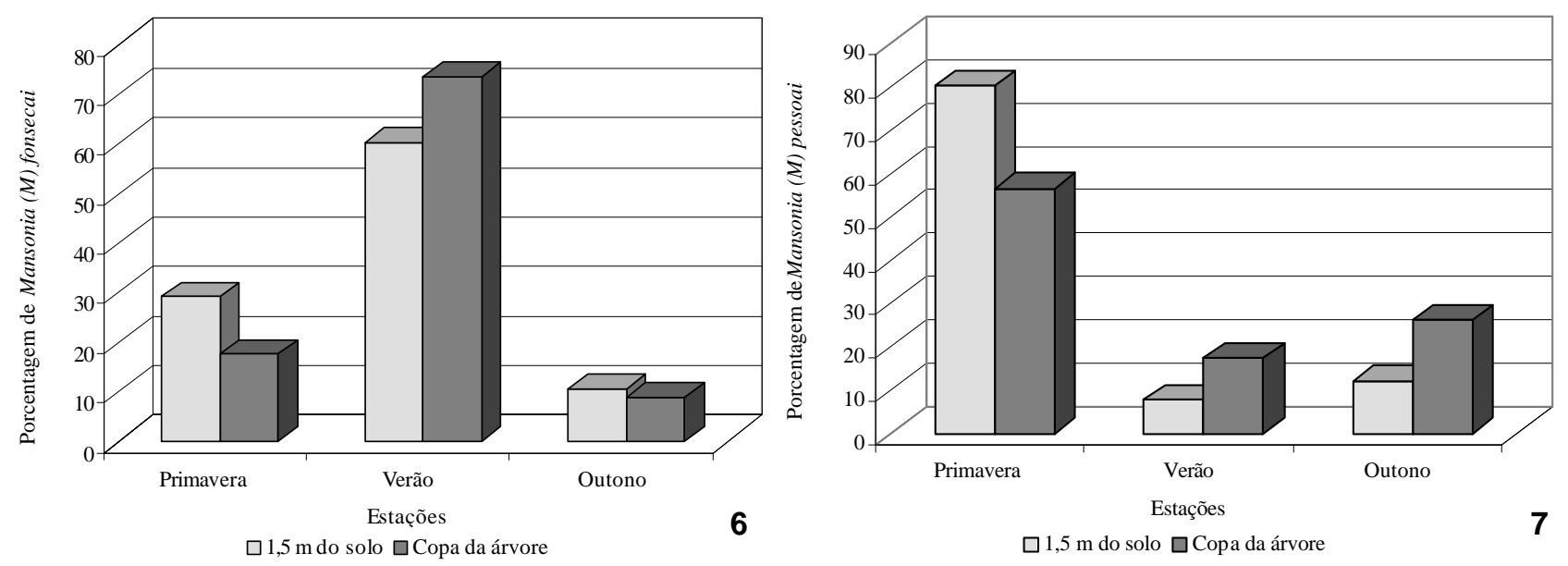

Figuras 6-7. Porcentagem de Mansonia (M ansonia) fonsecai (6) e M ansonia (Mansonia) pessoai (7) coletada na armadilha CDC-M iscada com mamífero, de setembro de 2000 a junho de 2001, em área de remanescente florestal do Parque Regional do Iguaçu, Curitiba, Paraná.

Tabela VI. Análise de regressão linear, exponencial e potencial dos dados climáticos de temperatura média, umidade relativa do ar e precipitação pluviométrica referentes aos meses em que foram efetuadas coletas de setembro de 2000 a junho de 2001, em área de remanescente florestal, do Parque Regional do Iguaçu, Curitiba, Paraná.

\begin{tabular}{|c|c|c|c|c|c|c|c|c|}
\hline \multirow{2}{*}{$r^{2}(-1 \leqslant r \leqslant 1)$} & \multicolumn{3}{|c|}{ Temperatura } & \multicolumn{3}{|c|}{ Umidade } & \multicolumn{2}{|c|}{ Precipitação pluviométrica } \\
\hline & Máxima & Mínima & Média & Máxima & Mínima & Média & Média & Somatória \\
\hline \multicolumn{9}{|l|}{ Linear } \\
\hline Culicidae & 0,067 & 0,041 & 0,065 & 0,113 & 0,324 & 0,249 & 0,147 & 0,184 \\
\hline CDC Ave & 0,137 & 0,100 & 0,141 & 0,319 & 0,327 & 0,379 & 0,568 & 0,582 \\
\hline CDC Mamífero & 0,138 & 0,100 & 0,142 & 0,322 & 0,331 & 0,384 & 0,564 & 0,576 \\
\hline \multicolumn{9}{|l|}{ Exponencial } \\
\hline Culicidae & 0,003 & 0,013 & 0,004 & 0,015 & 0,102 & 0,065 & 0,061 & 0,087 \\
\hline CDC Ave & 0,188 & 0,140 & 0,189 & 0,317 & 0,441 & 0,459 & 0,564 & 0,579 \\
\hline CDC Mamífero & 0,103 & 0,070 & 0,107 & 0,347 & 0,261 & 0,055 & 0,457 & 0,472 \\
\hline \multicolumn{9}{|l|}{ Potencial } \\
\hline Culicidae & 0,002 & 0,017 & 0,004 & 0,014 & 0,084 & 0,059 & 0,069 & 0,084 \\
\hline CDC Ave & 0,199 & 0,162 & 0,206 & 0,316 & 0,436 & 0,455 & 0,545 & 0,525 \\
\hline CDC Mamífero & 0,113 & 0,092 & 0,121 & 0,346 & 0,269 & 0,337 & 0,491 & 0,478 \\
\hline
\end{tabular}

do com ForatTINI (2002), os Mansoniini apresentam considerável poder de dispersão sendo considerados predominantemente exófilos e florestais, mostrando neste ambiente ligeira preferência para exercer atividade no solo.

Outros representantes de Culicidae também foram capturados. Culex foi o segundo gênero em número de exemplares, representado por $11 \%$ do total de culicídeos. A única espécie capturada Culex acharistus (Root, 1927), apresentou superioridade durante o verão, tanto para a armadilha CDC-M iscada com ave na copa da árvore, (67\%) quanto para a armadilha a $1,5 \mathrm{~m}$ do solo (62\%). Em armadilha iscada com mamífero cons- tatamos baixa freqüência desta espécie, similar ao resultado obtido por Almirón \& BREWER (1995) onde relataram, na Argentina, preferência desta espécie em al imentar-se em gal inhas do que em coel hos e tartarugas. A presença desta espécie no município de Curitiba, Paraná já havia sido relatada por Siıva \& LozOVE $(1996,1999)$ também em fragmento de mata inserido no tecido urbano (Tabs II e III, Fig. 4).

As demais espécies capturadas ocorreram em freqüência reduzida, independente da isca e estrato analisado, podendo reuni-las em dois conjuntos em relação ao habitat preferencial, ou seja silvestre e antrópico. 
Tabela VII. Análise de regressão linear, exponencial e potencial dos dados climáticos de temperatura média, umidade relativa do ar e precipitação pluviométrica referentes aos intervalos horários de seis dias que antecederam cada uma das quinze coletas, com armadilhas CDC-M, efetuadas de setembro de 2000 a junho de 2001, em área de remanescente florestal, do Parque Regional do Iguaçu, Curitiba, Paraná.

\begin{tabular}{|c|c|c|c|c|c|c|c|c|}
\hline \multirow{2}{*}{$r^{2}(-1 \leqslant r \leqslant 1)$} & \multicolumn{3}{|c|}{ Temperatura } & \multicolumn{3}{|c|}{ Umidade } & \multicolumn{2}{|c|}{ Precipitação pluviométrica } \\
\hline & Máxima & Mínima & Média & Máxima & Mínima & Média & Média & Somatória \\
\hline \multicolumn{9}{|l|}{ Linear } \\
\hline Culicidae & 0,005 & 0,021 & 0,035 & 0,004 & 0,029 & 0,042 & 0,000 & 0,000 \\
\hline CDC Ave & 0,137 & 0,140 & 0,125 & 0,057 & 0,017 & 0,001 & 0,002 & 0,002 \\
\hline CDC Mamífero & 0,057 & 0,108 & 0,135 & 0,000 & 0,066 & 0,067 & 0,000 & 0,000 \\
\hline \multicolumn{9}{|l|}{ Exponencial } \\
\hline Culicidae & 0,016 & 0,024 & 0,011 & 0,025 & 0,018 & 0,007 & 0,009 & 0,009 \\
\hline CDC Ave & 0,038 & 0,091 & 0,05 & 0,038 & 0,052 & 0,025 & 0,005 & 0,005 \\
\hline CDC Mamífero & 0,005 & 0,005 & 0,02 & 0,043 & 0,137 & 0,121 & 0,081 & 0,081 \\
\hline \multicolumn{9}{|l|}{ Potencial } \\
\hline Culicidae & 0,029 & 0,014 & 0,006 & 0,025 & 0,008 & 0,018 & 0,005 & 0,060 \\
\hline CDC Ave & 0,031 & 0,073 & 0,038 & 0,039 & 0,025 & 0,052 & 0,002 & 0,010 \\
\hline CDC Mamífero & 0,003 & 0,013 & 0,025 & 0,044 & 0,132 & 0,139 & 0,194 & 0,204 \\
\hline
\end{tabular}

Tabela VIII. Análise de regressão linear, exponencial e potencial dos dados climáticos de temperatura média, umidade relativa do ar e precipitação pluviométrica referentes aos intervalos horários de dezesseis dias que antecederam cada uma das quinze coletas, com armadilhas CDC-M, efetuadas de setembro de 2000 a junho de 2001, em área de remanescente florestal, do Parque Regional do Iguaçu, Curitiba, Paraná.

\begin{tabular}{|c|c|c|c|c|c|c|c|c|}
\hline \multirow{2}{*}{$r^{2}(-1 \leqslant r \leqslant 1)$} & \multicolumn{3}{|c|}{ Temperatura } & \multicolumn{3}{|c|}{ Umidade } & \multicolumn{2}{|c|}{ Precipitação pluviométrica } \\
\hline & Máxima & Mínima & Média & Máxima & Mínima & Média & Média & Somatória \\
\hline \multicolumn{9}{|l|}{ Linear } \\
\hline Culicidae & 0,003 & 0,000 & 0,002 & 0,008 & 0,152 & 0,124 & 0,065 & 0,066 \\
\hline CDC Ave & 0,129 & 0,160 & 0,154 & 0,106 & 0,021 & 0,019 & 0,000 & 0,000 \\
\hline CDC Mamífero & 0,046 & 0,027 & 0,048 & 0,057 & 0,274 & 0,226 & 0,091 & 0,091 \\
\hline \multicolumn{9}{|l|}{ Exponencial } \\
\hline Culicidae & 0,032 & 0,075 & 0,047 & 0,003 & 0,060 & 0,047 & 0,107 & 0,107 \\
\hline CDC Ave & 0,058 & 0,160 & 0,120 & 0,021 & 0,013 & 0,013 & 0,057 & 0,056 \\
\hline CDC Mamífero & 0,000 & 0,007 & 0,000 & 0,093 & 0,229 & 0,241 & 0,169 & 0,170 \\
\hline \multicolumn{9}{|l|}{ Potencial } \\
\hline Culicidae & 0,026 & 0,072 & 0,041 & 0,004 & 0,050 & 0,044 & 0,221 & 0,220 \\
\hline CDC Ave & 0,048 & 0,157 & 0,109 & 0,022 & 0,010 & 0,012 & 0,152 & 0,151 \\
\hline CDC Mamífero & 0,000 & 0,003 & 0,000 & 0,094 & 0,227 & 0,239 & 0,179 & 0,179 \\
\hline
\end{tabular}

No primeiro grupo temos: Phoniomyia quasilongirostris (Theobald, 1907) considerada como freqüentando preferencialmente a copa das árvores já tendo sido capturada sob hospedeiro humano (ForatTINI et al. 1989, GUIMARÃES et al. 1987, ForatTINI 2002); Coquillettidia venezuelensis (Theobald, 1912) considerada como possuindo atividade em diversas alturas do estrato arbóreo e preferência pelo hopedeiro cavalo para exercer a hematofagia (Lourenço-de-Oliveira \& Heyden 1986); Psorophora ferox (Humboldt, 1819) também freqüente em áreas florestadas, em relação à preferência por hospedeiro GomEs et al. (1987) obtiveram um grande percentual desta espécie alimentando-se no homem assim como SILVA \& LOZOVEI (1998); Aedeomyia squamipennis (Lynch Arribálzaga, 1878) tem seus imaturos coletados em local de água parada coberto com plantas aquáticas com incidência solar direta, enquanto as fêmeas adultas exercem a hematofagia em aves TEOdoro et al. (1994).

No segundo grupo, espécies que exploram ambiente antrópico temos: Ochlerotatus scapularis (Rondani, 1848), es- 
pécie considerada eclética na preferência pelo hospedeiro, exercendo a hematofagia sobre mamíferos e aves (LOURENÇO-DE-OLIVEIRA \& HEYDEN 1986, TEOdoRo et al. 1994); Ochlerotatus serratus (Theobald, 1901), alimentando-se preferencialmente no homem, com sua presença detectada no extra e peridomicílio apresentando pouca ou nenhuma tendência à domiciliação (ForattINI et al. 1987, GUIMARÃes et al. 1987, 2000, ForatTINI 2002); Limatus durhami (Theobald, 1901) considerada como dotada de capacidade de domiciliação, sendo capturada com grande freqüência na isca humana (GUIMARÃEs et al. 1987, ForaTtINI et al. 1989).

Outro representante do grupo das espécies de ambiente antrópico, Aedes al bopictus (Skuse, 1894), éconsiderada de grande importância epidemiológica. O primeiro registro desta espécie no Brasil foi em 1986 (Gomes et al. 1999) e de acordo com Miller \& BALLINGer (1988) a população introduzida demonstrou capacidade de transmitir dengue, febre amarela e vírus da encefalite eqüina venezuelana sob condições laboratoriais, porém Degallier et al. (2003) sugeriram que esta espécie ainda não pode ser considerada um vetor inter-humano durante epidemias de dengue no Brasil.

O fragmento de mata do Parque Regional do Iguaçu, através do método utilizado permitiu detectar a presença de espécies de Culicidae, com graus variados de importância epidemiológica e com tendência a explorar ambientes exófilos, florestais e peridomicilares, o que indica a necessidade de contínuo monitoramento desta população de dípteros de importância epidemiológica.

Podemos desenhar um quadro de preocupação, em conseqüência do caráter oportunista apresentado pelas espécies de Mansonia na utilização dos hospedeiros disponíveis, assim como as altas densidades alcançadas quando as condições ambientais são adequadas, no caso proliferação de macrófitas. Adicionando ainda, a potencialidade em transmitir arbovírus, o monitoramento das populações de Mansonia também deve receber atenção dos órgãos governamentais de saúde, frente aos novos cenários epidemiológicos.

\section{REFERÊNCIA S BIBLIOGRÁ FICAS}

Almirón, W.R. \& M.M. BreWer. 1995. Preferencia de hospedadores de Culicidae (Diptera) recolectados en el centro de la Argentina. Revista de Saúde Pública, São Paulo, 29 (2): 108-114.

Christensen, B.M. \& W.M. AndreWs. 1976. Natural infection of Aedes trivittatus (Coq.) with Dirofilaria immitis in Central Iowa. The Journal of Parasitology, Lawrence, 62 (2): 276280.

Degallier, N.; J.M.S. Teixeira; S. da S. Soares; R.D. Pereira; S.C.F. Pinto; A. de J.M. Chaib; P.F.C. Vasconcelos \& E. Oliveira. 2003. Aedes albopictus may not be vector of dengue virus in human epidemics in Brazil. Revista de Saúde Pública, São Paulo, 37 (3): 386-387.
Forattinı, O.P. 2002. Culicidologia Médica. Identificação, Biologia, Epidemiologia. São Paulo, Editora da Universidade de São Paulo, vol. 2, 860p.

Forattini, O.P.; A. DE C. Gomes; D. Natal; I. Kakitani \& D. MaruCCI.1987. Preferências alimentares de mosquitos Culicidae no Vale do Ribeira, São Paulo, Brasil. Revista de Saúde Pública, São Paulo, 21 (3): 171-187.

Forattinı, O. P.; A. DE C. Gomes \& I. KaKITANı. 1989. Observações sobre mosquitos Culicidae adultos em cultivo irrigado de arroz no Vale do Ribeira, Estado de São Paulo, Brasil. Revista de Saúde Pública, São Paulo, 23 (4): 307-312.

Forattini, O.P.; I. Kakitani; G.R.M. Marques \& M. de Brito. 1998. Formas imaturas de anofelíneos em recipientes artificiais. Revista de Saúde Pública, São Paulo, 32 (2): 189-191.

Gad, T.M.; H.A. Farid; R.R.M. RamzY; M.B. RIaD; S.M. Presley; S.E. COPE; M.M. HASSAN \& A.N. HASSAN. 1999. Host feeding of mosquitoes (Diptera: Culicidae) associated with the Recurrence of Rift Valley Fever in Egypt. Journal of Madical Entomology, Lanham, 36 (6): 709-714.

Gilliland, T.M.; W.A. Rowley; N.S. SWack; J.K. Vandyk \& M.G. BARTOCES. 1995. Arbovirus Surveillance in lowa, USA, during the flood of 1993. Journal of the American Mosquito Control Association, Lakes Charles, 11 (2): 157-161.

Gomes, A. DE C.; E.X. Rabello \& D. NatAl. 1985. Uma nova câmara coletora para armadilha CDC - miniatura. Revista de Saúde Pública, São Paulo, 19: 190-191.

Gomes, A de C; O.P Forattinı \& D. Natal. 1987. Composição e atividade de mosquitos Culicidae.Emprego de Armadilha CDC no Vale do Ribeira, Estado de São Paulo, Brasil. Revista de Saúde Pública, São Paulo, 21 (5): 363-370.

Gomes, A.de C.; M.D. Bitencourt; D. Natal; P.L.S. Pinto; L.F. Muccl; M.B. de Paula; P.R. Urbinatti \& J.M.S. Barata. 1999. Aedes albopictus em área rural do Brasil e implicações na transmissão da febre amarela silvestre. Revista de Saúde Pública, São Paulo, 33 (1): 95-97.

Guimarães, A.E.; M. Arlé \& R.N.M. Machado. 1987. Mosquitos no Parque Nacional da Serra dos Órgãos, Estado do Rio de Janeiro, Brasil. IV. Preferência Alimentar. Memórias do Instituto Oswaldo Cruz, Rio de Janeiro, 82 (2): 277-285.

Guimarães, A.E.; C. Gentile; C.M. Lopes; A. Sant'Ana \& A.M. Jovita. 2000. Ecologia de mosquitos (Diptera, Culicidae) em áreas do Parque Nacional da Serra da Bocaina, Brasil. I - Distribuição por habitat. Revista de Saúde Pública, São Paulo, 34 (3): 243-250.

Lourenço-De-Oliveira, R. \& R. Heyden. 1986. Algunsaspectos da ecologia dos mosquitos (Diptera, Culicidae) de uma área de planície (Granjas Calábria), em Jacarepaguá, Rio de Janeiro. IV - Preferências alimentares quanto ao hospedeiro e freqüência domiciliar. Memórias do Instituto Oswaldo Cruz, Rio de Janeiro, 81 (1): 15-27.

MAACK, R. 1981. Geografia Física do Estado do Paraná. Rio de Janeiro, José Olympio, 2ae ed., 450p.

MACIÁ, A. 1996. Variación intraespecífica del tamaño de adul- 
tos de Culicidae (Diptera) en el área Platense, Provincia de Buenos Aires, Argentina. Acta Entomologica Chilena, Santiago, 20: 63-70.

Marquetti, M. del C.; A. Navarro; J.A.B. Lazcano \& F.A. Garcia. 1990. Comparacion de 3 metodos de captura de anofelinos em uma zona com riesgo de transmission paludica. Revista Cubana de Medicina Tropical, Habana, 42 (2): 247-253.

MilleR, B.R. \& M.E. BALLInger. 1988. Aedes al bopictus mosquitoes introduced into Brazil: vector competence for yellow fever and dengue viruses. Transactions of the Royal Society of Tropical Medicine and Hygiene, London, 82: 476-477.

Natal, D.; D. Maruccl; I. M. dos Reis \& E.A.B. Galati.1991. Modificação da armadilha CDC com testes para coletas de Flebotomíneos (Diptera) Revista Brasileira de Entomologia, Curitiba, 35 (4): 697-700.

Pereira, L.E.; A. Suzuki; T.L. Coimbra; R.P. de Souza \& E.L.B. Chamelet. 2001. Arbovírus Il héus em aves silvestres (Sorophila caerulescens e M olothrus bonariensis). Revista de Saúde Pública, São Paulo, 35 (2): 119-123.

Silva, M.A.N. DA \& A.L. Lozoveı. 1996. Criadouros de imaturos de mosquitos (Diptera, Culicidae) introduzidos em mata preservada na área urbana de Curitiba, Paraná, Brasil. Revista Brasileira de Zoologia, Curitiba, 13 (4): 1023-1042. . 1998. Mosquitos (Diptera, Culicidae) capturados com isca humana em área preservada de Curitiba, Paraná. Revista Brasileira de Zoologia, Curitiba, 15 (4): 965-976.

Recebido em 02.XII.2003; aceito em 09.XI.2004.
1999. Ocorrência de Haemagogus (Conopostegus) leucocelaenus (Dyar \& Shannon) eToxorhynchites (Rynchiella) theobaldi (Dyar \& Knab) em ocos de árvores em Capão de mata, Curitiba, Paraná, Brasil. Revista Brasil leira de Zoologia, Curitiba, 16 (1): 257-267.

Sudia, W.D. \& R.W. Chamberlain. 1962. Battery-operated light trap, in a improved model. Mosquito News, Fresno, 22 (22): 126-129.

Teodoro, U.; A.L.F. Guilherme; A.L. Lozovel; V.L.S. Filho; A.A. Sampaio; R.P. Spinosa; M.E.M.C. Ferreira, O.C. Barbosa \& E.M. DE LIMA. 1994. Mosquitos de ambientes peri e extradomiciliares na região sul do Brasil. Revista de Saúde Pública, São Paulo, 28 (2): 107-115.

Talpe-Lagos, C.B. \& D. Natal. 2003. Abundância de culicídeos em área metropolitana preservada e suas implicações epidemiológicas. Revista de Saúde Pública, São Paulo, 37 (3): 275-279.

Vasconcelos, P.F. da C.; J.F.S. Travassos da Rosa; A.P.de A. Travassos da Rosa; N. Dégallier; F. de P. Pinheiro \& G.C. Sá Filho. 1991. Epidemiologia das encefalites por arbovírus na Amazônia brasileira. Revista do Instituto de Medicina Tropical, São Paulo, 33 (6): 465-476.

ZaIm, M.; ERShadI, M.R.Y.; A.V. ManouchehrI \& M.R. Hamdi. 1986. The use of CDC light traps and other procedures for sampling malaria vectors in southern Iran. Journal of the American Mosquito Control Association. 2 (4): 511-515.

Revista Brasileira de Zoologia 21(4): 877-886, dezembro 2004 